\title{
Gender-Sternchen, Binnen-I oder generisches Maskulinum, ... (Akademische) Textstile der Personenreferenz als Registrierungen?*
}

\author{
Helga Kotthoff (Freiburg)
}

\begin{abstract}
For more than 40 years, a debate on gender-related person references has been taking place in the German-speaking world. My contribution starts with a differentiation of four registers, which are currently practiced in writing and have developed specific reasoning discourses and specific social contexts of usage, as I try to show. I am going to examine these four styles of gendered person reference as "registers" in the sense of anthropological linguistics (Agha 2007). This concept of "enregisterment" implies that the producers connect themselves to a socio-symbolic cosmos and can be perceived with cultural evaluations (in production and reception), for example, as conservative, feminist, queer, liberal (Kotthoff 2017). Here I shall explore their (socio)linguistic underpinnings within conceptions of language ideology in order to grasp the communication-reflexive charges of these discourses.
\end{abstract}

\section{$1 \quad$ Einleitung}

Seit über 40 Jahren findet im deutschsprachigen Raum eine Debatte um geschlechterbezogene Personenreferenzen statt. Mein Beitrag setzt bei einer Binnendifferenzierung von vier Registern an, die sich inzwischen mit spezifischen sozialen Verortungen herausgebildet haben, was ich $\mathrm{zu}$ zeigen versuche. Die tradition elle Schreibpraxis mit einem generisch gemeinten, geschlechtsübergreifenden Maskulinum ${ }^{1}$ (Typ 1), wie sie etwa von Eisenberg (2017), Glück (2018) oder dem Verein für Deutsche Sprache (VDS) vertreten wird, argumentiert strukturalistisch-systemgrammatisch für eine durchgängige Generizität des auf Personen referierenden Maskulinums. Sie hält Gendern (mit Diewald/Steinhauer 2017 wurde dieses Verb und seine Nominalisierung für gendersensible Schreibungen akzeptabilisiert) für unnötig.

Trömel-Plötz (1978) und Pusch (1979: 1984) beschrieben die Ambiguität des Maskulinums für viele Kontexte als nahegelegte Referenz auf männliche Wesen und das Verschwinden weiblicher Personen in der mentalen Repräsentation. Bis heute zeigen psycholinguistische und kog-

\footnotetext{
* Ich danke den Teilnehmer/innen an der Tagung in Cluj-Napoca „Dominanz, Macht, Sprache“ 2019 und denjenigen an der Tagung „Reflexivität kommunikativer Praktiken“ an der Universität Hamburg 2019 und Peter Auer, Evelyn Ferstl und Lars Bülow für Diskussionen.

${ }^{1}$ Eine generische Personenbezeichnung bezieht sich abstrakt auf eine Gattung und nicht-identifizierte Personen (cf. Petterson 2011; Kotthoff/Nübling/Schmidt 2018: 92). Das linguistische Konzept von Generizität ist enger als dasjenige aus dem öffentlichen Diskurs.
} 
nitionspsychologische Experimente immer wieder, dass Frauen eher mitgedacht werden, wenn die syntaktisch-semantische Struktur die explizite Information enthält, dass neben Männern auch auf Frauen Bezug genommen wird (Scheele/Gauler 1993; Stahlberg/Szessny 2001; Ferstl/Kaiser 2013). Diese Kritik generierte eine feministisch inspirierte personenreferentielle Praxis (Typ 2). Weiter unten erläutern wir Strategien der Beidnennung, der Kurzschreibung der Beidnennung mit Binnen-I und der Neutralisierung, beispielsweise durch Komposita mit -kraft (Lehrkraft). Typ 2 und Typ 1 konturierten sich in Kontroversen mit- und aneinander.

Im teilweisen Gegensatz zu Typ 1 und auch zu einigen Strategien der feministisch inspirierten Sprachpraxis (Typ 2) argumentieren Vertreter/innen einer queeren Sicht (Typ 3), dass Beidnennungen oder Binnenmajuskeln die Dichotomie der Geschlechtszuordnung stärken, statt referentielle Geschlechtervielfalt herzustellen. Um explizite Feminisierung zu vermeiden, werden hier (wie seit Langem auch bei Typ 2) Neutralformen praktiziert (z. B. Partizipien wie Studierende) und neuerdings noch neue Zeichenintegrationen an Morphemgrenzen zur Movierung bei Personenreferenzen vorgeschlagen, speziell der Unterstrich und der Asterisk (auch „Genderstern“) (z. B. Lehrer_innen, Bäcker*innen, Typ 3). Gemäß Hornscheidt (2012) und Baumgartinger (2008) sollen beide Grapheme hauptsächlich zu einer kognitiven Repräsentation von Personen mit nicht-binären Geschlechtsidentitäten einladen (aber es gibt auch viele vagere Begründungen in Richtung sexueller Identität, die weiter unten aufgeführt werden). Diese Sprachreform rekonturiert die feministisch inspirierten Vorschläge der neunziger Jahre (hier Typ 2) mit Begründungen, die ich weiter unten einer kritischen Betrachtung unterziehe (wie auch andere Schreibpraktiken und ihre Begründungen).

Daneben haben sich in einigen Medien (z. B. bei Radiosendern und einigen Tageszeitungen) oder auch in den Sozialwissenschaften Stile „flexiblen Genderns“ (Typ 4) herausgebildet, die oft mit Neutralformen und nur punktueller Beidnennung der Geschlechter arbeiten.

Wie es in der internetbezogenen Schreibstilforschung inzwischen bereits Tradition hat (cf. Spitzmüller 2015; Busch 2018), möchte ich auch diese genderbezogenen Textpraktiken im anthropologisch-linguistischen Lichte (cf. Agha 2007) mit ihren metapragmatischen Komponenten betrachten und kritisch diskutieren, ob und wie man im Rahmen dieser anthropologischlinguistischen Ansätze die kommunikative Reflexivität und Ideologiepotentiale fassen könnte.

Im Aufruf zu einer Tagung an der Universität Hamburg schreiben Busch/Droste/Wessels (2019), dass kommunikative Praktiken immer auch auf sich selbst und ihre Verwendungs-zusammenhänge verweisen und dass RezipientInnen auf sozial geteilte Interpretationsrahmen schließen können. Zu einigen Praktiken entwickeln sich Metasprachdiskurse und genau solche laufen im deutschsprachigen Raum öffentlich rund um das sogenannte „Gendern“ ab. Darum soll es gehen.

\section{Sprachideologien und Registrierungen}

Vor allem die interaktionale Soziolinguistik und die anthropologische Linguistik (cf. Gumperz 1992) gehen davon aus, dass Unterscheidungen im Sprachgebrauch symbolisch genutzt werden, um Gemeinschaften rund um Geschichten des Gebrauchs zu formen, die in bestimmten kommunikativen Praktiken sedimentieren und dann zur Identitätsanzeige genutzt werden können. Zentral dafür sind nach Agha $(2003 ; 2007)$ Prozesse der Enkodierung (enregisterment) - 
soziohistorische Prozesse, durch die spezielle Formen semiotischen Verhaltens in „kulturelle Modelle" transformiert werden, das heißt in Formen, die auf Stereotypen verweisen. Spezifische semiotische Unterscheidungen werden als Register (register) konventionalisiert und als solche mit ihren sozialen Zuordnungen erkennbar (Droste 2014; 2017). Gegenwärtig praktizierte Stile des sog. „Genderns“ (Diewald/Steinhauer 2017) stelle ich hier als Register vor. Ob und in welchem Ausmaß solche Register sprachideologisch unterfüttert werden, ist die Hauptfrage meines Beitrags. Nach Silverstein (2003:193) (auf den sich auch Spitzmüller 2005: 254 beruft) sind Sprachideologien "sets of beliefs about language articulated by users as a rationalisation or justification of perceived language structure and use."

In der Tradition von Antonio Gramsci (1991) wird der common sense als ideologisch unterfüttert angesehen. Im Bezug auf Gender und Personenreferenz wurde der akzeptierte common sense eines auch geschlechterübergreifend gemeinten Maskulinums in den letzten 40 Jahren erschüttert (weiter unten mehr dazu). Seither laufen Enkodierungen ab.

Van Dijk (1995) grenzt sich vom Marxschen Ideologiebegriff des „notwendig falschen Bewusstseins“ ab. Statt eine irgendwie geartete Falschheit zu veranschlagen, unterstellt er eher Funktionalität für Gruppeninteressen, die mit mehr oder weniger Macht verbreitet werden können. Van Dijk betont den engen Zusammenhang von Kognition und sozialer Erfahrung:

Ideologies in our perspective are not merely "systems of ideas", let alone properties of the individual minds of persons. Neither are they vaguely defined as forms of consciousness, let alone "false consciousness". Rather, they are very specific basic frameworks of social cognition, with specific internal structures, and specific cognitive and social functions.

(van Dijk 1995: 21, zitiert nach Blommaert 2005: 162)

Blommaert (2005:165f.) fasst weitere Ansätze zusammen, so die von Louis Althusser und Pierre Bourdieu, die die materielle und institutionelle Verankerung von Ideologien betonen, die zum legitimen Wissen werden können und mehr oder weniger umfassend und hegemonial ausfallen. Bourdieu (1983) betont in allen seinen Stiluntersuchungen den Distinktionsfaktor, der für die hier betrachteten Registrierungen sehr bedeutsam ist.

Hinter Typ 1 steht die Macht der Tradition, verschiedener Institutionen wie beispielsweise des deutschen Strafrechts ${ }^{2}$ und literarischer Werke. ${ }^{3}$ Hinter den Praktiken von Typ 2 standen universitäre Schreibstile in geistes- und sozialwissenschaftlichen Bereichen, Gremien von Universitäten, deren Gleichstellungsstellen dahingehende Vorschläge unterbreitet hatten und ähnlich die einiger Städte, feministische Organisationen, linke Zeitungen und pädagogische und künstlerische Einrichtungen. Für Typ 3 sind dergleichen Bemühungen stark im Gange (Stadt Hannover, ${ }^{4}$ einige universitäre Leitfäden, Kampagnen der Internet-Plattform PINKSTINKS, Prakti-

\footnotetext{
${ }^{2}$ Das deutsche Strafgesetzbuch und andere Gesetzestexte verwenden das generische Maskulinum, was bedeutet, dass mit der Bezeichnung „Minister“ auch Ministerinnen und mit der Bezeichnung „Mörder“ auch Mörderinnen gemeint sind, sie werden mit eingeschlossen. Ferner ergeben sich aus der regelmäßigen Verwendung männlicher Personenbezeichnungen Formulierungen wie „der Verlobte des Beschuldigten“ ( 52 Abs. 1 Nr. 1 StPO).

${ }^{3}$ Beim WDR 5 Funkhausgespräch „Das Gendersternchen - Unfug oder Fortschritt?“ vom 13.06.2019 hält Jagoda Marinic fest, dass in literarischen Texten fasst gar nicht gegendert wird. Das entspricht meiner eigenen Lektüreerfahrung.

${ }^{4}$ Die neue Empfehlung ist für sämtlichen Schriftverkehr der Verwaltung der Stadt Hannover verbindlich. Sie wird schrittweise in E-Mails, Präsentationen, Broschüren, Presseartikeln, Drucksachen, Hausmitteilungen, Flyern,
} 
ken der Gruppierung „Fridays for Future“...) und lösen Typ 2 ab. Typ 2 und 3 sind besonders in linken ${ }^{5}$ und intellektuellen Milieus zu Hause. Den Typen 1, 2 und 3 können wir Verankerungen in Institutionen zuschreiben. Typ 3 grenzt sich von Typ 1 und Typ 2 gleichermaßen ab. Typ 2 wurde und Typ 3 wird derzeit der Öffentlichkeit in zahlreichen Neuauflagen von Richtlinien unterbreitet. ${ }^{6}$ Genau diese Bemühungen, über Richtlinien Schreibweisen zu vereinheitlichen, rufen nicht nur Vertreter/innen des Typ 1 auf den Plan.

Irvine/Gal (2000: 37f.) unterscheiden als analytischen Rahmen für die qualitative Untersuchung von Sprachideologien drei semiotische Prozesse, in welchen sich die Sprachideologie niederschlägt: Ikonisierung (iconization), Rekursivität (fractal recursivity) und Löschung (erasure), die bereits Androutsopoulos (2006) als analytischer Leitfaden für seine Untersuchung von Internet-Unterhaltungsgattungen und metasprachlichen Medienberichten zu Ethnolekten dienen. Im Bezug auf die Varietätengefüge einer Sprache sprechen Irvine/Gal (2000:37f.) z. B. von Löschung, wenn die Standardvarietät vor dem Hintergrund einer Homogenitätsannahme beispielsweise als die (z. B.) Sprache bezeichnet wird. Sie nehmen die Sprachideologie von Sprecher/innen des Serbischen bezüglich des Mazedonischen als Beispiel. Sie ikonisieren die einfachere nominale Morphologie des Mazedonischen als typisch für "uncultivated country bumpkins" (ibd.: 69). Irvine und Gal erklären, dass durch eine solche Ikonisierung eine Wahrnehmung befördert würde, das Mazedonische "had no grammar," was einer Aufwertung des Serbischen dienlich sei. Die Komplexitäten des mazedonischen Verbsystems bleiben dann außen vor (ibd.); ein simples Modell der Gegenüberstellung siegt. Unter Ikonsisierung werden soziale Assoziationen zwischen Sprachmerkmalen und deren typischen Verwender(inne)n verstanden. Solche Zuordnungen betreffen immer auch „fraktal rekursiv“ das mitkonstruierte Gegenteil (beispielsweise als Stadt/Land-Kontrast oder als fortschrittlich/rückschrittlich-Kontrast). Betont werden „loadings of moral and political interests” (Irvine 1989), mit denen ein Register ausgestattet wird. Dies ist im vorliegenden Themenbereich sehr auffällig, allerdings nicht überall gleich dick aufgetragen. Wir halten hier fest, dass zur Bestimmung von Ideologiehaftigkeit sowohl Grade an Wissenschaftsferne als auch an Explizitheit und Forcierung des Moralisierens von Bedeutung sind. Typ 4 des flexiblen Genderns, also die nicht durchgängige Beidnennung wird in vielen Artikeln des Schweizerischen Tagesanzeigers, der Frankfurter Rundschau und der Süddeutschen Zeitung praktiziert, auch beispielsweise in vielen Hörtexten des Senders Österreich 1 und des Schweizer Radios SRF2. Diese Praxis verzichtet weitgehend auf eigene Begründungen; es gibt dazu bis dato keine Metadiskurse. Der Deutschlandfunk gendert hingegen seit etwa 6 Monaten durchgängig mit unterschiedlichen Strategien. ${ }^{7}$

\footnotetext{
Briefen, Formularen und Rechtstexten umgesetzt. Die wichtigste Grundregel ist, überall da, wo es möglich ist, geschlechtsumfassende Formulierungen zu verwenden.

${ }^{5}$ Innerhalb der Linken werden allerdings unterschiedliche Positionen vertreten. Die deutsche Partei „Die Link““ verzichtet in Internet-Publikationen auf Gendern, um Leseprozesse zu erleichtern, z. B. für Menschen mit eingeschränkten Kompetenzen (Lehmann 2019).

${ }^{6}$ Z. B. Die Gleichstellungsbeauftragte der Universität zu Köln (2017): ÜberzeuGENDERe Sprache. Siehe auch Kolek (2019).

${ }^{7}$ Dazu Ann-Kathrin Büüsker, eine Vertreterin des Deutschlandfunks, in einem Interview der taz zum Thema „Inklusive Sprache in den Medien: Intuition und Abwehr“ vom 07.06.2020. Zu den Strategien gehören auch Pluralreferenzen mit Glottisschlag vor dem Femininmorphem.
}

ISSN 1615-3014 


\subsection{Systemlinguistik}

Gauger (2017: 72f.) fasst die systemlinguistische Position zur Personenreferenz so zusammen:

Unsere Sprache hat sich, wie sehr viele andere Sprachen, für die männliche Form entschieden, wenn es um das Generische geht, wenn also vom Geschlecht abgesehen wird: „Lehrer werden zu schlecht bezahlt" oder „Dem Franzosen ist gutes Essen wichtiger als dem Deutschen“ oder „Die Zahl der Radfahrer nimmt zu.“ Aber natürlich ist es Unsinn zu sagen, die Sprache habe sich „entschieden" (man sagt halt so), denn eine Sprache entscheidet sich nicht - es hat sich in ihr eben so ergeben: historisch. Dies heißt nun aber, dass Wörter wie Lehrer, Franzose, Deutscher, Radfahrer [...] zwei Bedeutungen haben: erstens die allgemeine, generische, geschlechtlich undifferenzierte, zweitens das männliche Exemplar.

Gauger (2017: 72f.)

Man rede sprachwissenschaftlich auch von einer „,inklusiven Opposition“: Lehrer stehe sprachlich ,in Opposition“ zu allen anderen Berufen, enthalte dann aber in sich selbst auch die ganz andere Opposition zu Lehrerin; in die Opposition zu allen anderen Berufen sei also die Opposition zu Lehrerin eingeschlossen, so dass Lehrer auch für Lehrerin stehen könne, nicht aber Lehrerin für Lehrer (Gauger 2017: 73). Das heißt „Femininmotion.“ Diese gilt als markierter wegen ihres „Mehr“ an Kodierung. Haspelmath (2006) meint, die meisten Asymmetrien, die sich in Sprachen finden lassen, könnte man statt über die Markiertheitstheorie besser unter Rückgriff auf die Frequenz der Opponenten erklären. Er untersucht Kontexte, in denen der Terminus „Markiertheit“ beziehungsweise „markedness“ in linguistischen Forschungstexten verwendet wird. Er findet den Terminus zu polysem und macht zwölf verschiedene Bedeutungen von „markedness“ aus. Er subsumiert sie unter vier Oberbegriffe: „Markedness as complexity, as difficulty, as abnormality, and as a multidimensional correlation“. Komplexität einer Kodierung heißt etwa, dass der Plural mittels mehr sprachlichen Materials kodiert wird als der Singular. Dem entspricht auch das Femininum. Er plädiert dafür, eher von „offen und sichtbar kodiert“ zu sprechen. Anstatt auf das Konzept der Markiertheit zurückzugreifen, durch die eine Äußerung komplexer oder einfacher für das menschliche Gehirn wird, solle man von frequenzbedingten Faktoren ausgehen. Den Faktor einer hohen „Gebrauchsfrequenz" hatte traditionell das Maskulinum auf seiner Seite. Das könnte sich dadurch ändern, dass Frauen im öffentlichen Leben heute insgesamt sichtbarer würden (Haspelmath 2019).

Lewandowski (1994: 353) schreibt in der 5. Auflage unter der Rubrik „Genus“:

Grammatisches Geschlecht; -grammatische Kategorie, Merkmal von Substantiv, Artikel, Adjektiv, Pronomen, das sich im allgemeinen von seiner Bindung an das natürliche Geschlecht fast ganz gelöst hat.

Lewandowski (1994: 353)

Im Unterschied zu dieser bis dato in der Linguistik gängigen Loslösungshypothese von Genus und Sexus enthält bereits die Duden-Grammatik von 1998 (Duden-Grammatik 1998: 200) im Unterkapitel zum Genus der Substantive den Passus: 
2. Besonders bei Berufsbezeichnungen und Substantiven, die den Träger bzw. die Trägerin eines Geschehens bezeichnen (Nomina Agentis), wird die Verwendung des generischen Maskulinums immer mehr abgelehnt. Bei Bezug auf weibliche Personen werden häufig feminine Formen (z. B. auf -in oder entsprechende Umschreibungen) verwendet.

(Duden-Grammatik 1998: 200)

Hier wird bereits deutlich, dass sich die Duden-Redaktion von Debatten rund um einen Nexus von Genus und Sexus im Bereich von Personenreferenz (Lobin/Nübling 2018) inspirieren ließ. 2016 findet sich in der Duden-Grammatik (2016: 157) ein breites Eingehen auf das Thema. Er unterscheidet

- sexusindifferent

- spezifisch männlich

- spezifisch weiblich

Und Genderdebatten werden aufgegriffen:

Eigentlich müsste hier noch weiter differenziert werden. Man kann fragen, in wieweit rein biologische Merkmale eine Rolle spielen (-Sexus im engen Sinne, biologisches oder ,natürliches" Geschlecht) oder aber soziale und psychologische Merkmale (-Gender, soziales und psychologisches Geschlecht). Bei der sozialen Kategorie Gender ist vieles im Fluss [...]

Dann werden substantivische Personenbezeichnungen in Klassen unterteilt:

- Klasse A sexusindifferent (wie der Star, die Person)

- Klasse B sexusspezifisch (wie der Junge, die Dame)

- Klasse C umfasst maskuline Personenbezeichnungen, die sowohl sexusspezifisch (Bezug nur auf Männer) als auch sexusindifferent gebraucht werden. Neben solchen Maskulina steht gewöhnlich eine feminine Ableitung, die sexusspezifisch auf weibliche Personen referiert (Klasse B), meist mit dem Suffix - in (trad. Fachausdruck Movierung).

(ibd.: 160)

\subsection{Psycholinguistische und kognitionspsychologische Experimentalstudien}

Viele Experimentalstudien zeigen, dass die Ambiguität maskulin-geschlechtsübergreifender Referenzen in vielen Kontexten weibliche Personen in der mentalen Repräsentation zurücktreten lässt (cf. Kotthoff/Nübling/Schmidt 2018:95f.). Mittlerweile liegen ca. 20 (psycho-) linguistische Untersuchungen zur Überprüfung dieser Maskulina vor. Über unterschiedliche Methoden und Designs wird in (mündlichen wie schriftlichen) Rezipiententests und Reaktionszeitexperimenten der Frage nachgegangen, ob Maskulina wie Einwohner, Tourist, Lehrer ausschließlich, mehrheitlich oder paritätisch männliche Assoziationen evozieren (zu einem Forschungsüberblick cf. Kotthoff/Nübling/Schmidt 2018: 91-127). Sämtliche Untersuchungen weisen in die gleiche Richtung: Das generische Maskulinum ruft mehr Männer als Frauen auf und die Verarbeitung eines weiblich referierenden Maskulinums beansprucht deutlich mehr Zeit als die eines männlich referierenden (cf. Braun et al. 1998; Stahlberg/Sczesny 2001; Gygax et al. 2008). Die Tests zeigen, dass maskuline Formen gerade im Singular nicht geschlechterübergreifend verstanden werden, und dass im Deutschen die grammatische Genuszuweisung die wissensbasierte Stereotyp-Information zumindest beeinflussen kann (cf. Gygax et al. 2008). Diese Befunde bestätigen, dass maskuline Personenreferenzen selten geschlechtsübergreifend 
verstanden werden, auch wenn produzentenseitig „Frauen mitgemeint“ sein mögen. Trotz unterschiedlicher Designs sind die Ergebnisse der Experimente zwar kohärent, doch wurden einige Faktoren, die die Geschlechtswahrnehmung beeinflussen, bislang nicht systematisch untersucht. Solche Faktoren sind z. B. die Numeruswahl des Nomens (der vs. die Politiker) oder ob das Nomen eine Berufs- oder eine Rollenbezeichnung darstellt (Politiker vs. Einwohner), wie stereotyp seine Semantik ist (Pilot vs. Sachbearbeiter vs. Pfleger), und schließlich, in welchem thematischen und lebensweltlichen Kontext die Sätze stehen. Außerdem wurde bis dato nur die Rezeption von Kurztexten erforscht. Wie sich beispielsweise randomisierter Genuswechsel (Mischung sog. generischer Maskulina und Feminina) in längeren Lese- oder Hörtexten auswirkt, ist derzeit ebenso wenig bekannt wie alle anderen Strategien eines Wechsels von Personenreferenzen (Wechsel von generischem Maskulinum, Beidnennung oder auch unterschiedliche Kurzschreibungen einer Beid- und/oder Gendervielfaltnennung ${ }^{8}$ ).

\subsection{Das Übliche wird konservativ}

Diewald/Steinhauer (2017) haben in ihrem Ratgeber „Richtig gendern“ dem Verb „gendern“ für alle Verfahren einer geschlechterfairen Sprachgestaltung eine neutrale Konnotation zugeordnet. Sie graduieren die Genderrelevanz sprachlicher Einheiten im Text (ibd: 72f.). Sie unterscheiden spezifische Referenz (Susanne ist unsere Dozentin), bei der eine korrekte Geschlechtszuweisung notwendig ist, und nichtspezifische Referenz (Jeden Tag läuft mir ein Postbote über den Weg), bei der sie diese auch empfehlen. Mäßige Genderrelevanz sehen sie z. B. bei klassenbezogenen Referenzen, wie sie etwa für Kollektiva gilt (z. B. Ableitungen auf -schaft).

Linguisten wie Eisenberg (2017) und Bayer (2019) halten feminine Ableitungen nur bei konkreter Referenz und Ansprache für nötig. Ansonsten argumentieren sie u. a. mit Jakobsons Markiertheitstheorie:

Jakobsons Argument überträgt sich ohne Einschränkungen auf das Deutsche. „Student“ und „Stu-
denten“ bedeuten keine Festlegung auf das natürliche Geschlecht und somit auf männliche We-
sen. Diese Substantive sind ,unmarkierte“ Formen, die den Bezug auf weibliche Wesen, die stu-
dieren, automatisch mit einschliessen. Erst wenn man betonen will, dass man sich ausschliesslich
auf die weibliche Spezies beziehen möchte, kommen „Studentin“ und „Studentinnen“ zum Ein-
satz. Es gibt demnach, folgt man der unbestrittenen linguistischen Argumentation von Roman
Jakobson, keinen Grund, das gute alte Studentenwerk in ein Studierendenwerk umzutaufen.

(Bayer 2019)

In den Artikeln der systemgrammatisch argumentierenden Linguist(inn)en wird nicht auf Arbeiten eingegangen, die die Markiertheitstheorie in ihre Schranken verweisen (cf. dazu Haspelmath 2006) und auch nicht auf psycholinguistische und kognitionslinguistische Forschung, die anhand von Kurztexten zeigt, dass maskuline, genderübergreifend gemeinte Referenzen stärker an Männer denken lassen (siehe das nächste Unterkapitel). Das Ausblenden solcher Studien dient einer Homogenisierung der eigenen Sicht auf Sprachpraktiken und steht somit hier unter dem Ideologieverdacht einer Verengung.

\footnotetext{
${ }^{8}$ Damit meine ich Schreibungen, die über besondere Grapheme entweder Referenzen auf genderdiverse Personen herzustellen beabsichtigen oder auf weitere Identitätszuschreibungen.
}

ISSN 1615-3014 
Textpraktiken, in deren Hintergrund solche Annahmen walten, finden sich in den meisten Artikeln in überregionalen Tageszeitungen wie Die Welt und Frankfurter Allgemeine Zeitung und in regionalen wie Der Südkurier oder Westfalenpost. Auch in vielen Geschäftsbereichen (z. B. Banken) herrscht dieses Register vor. Da Teile der Öffentlichkeit die geschlechterübergreifende Gemeintheit der maskulinen Referenz in Zweifel ziehen, kommt es immer wieder zu Kampagnen wie z. B. der von „PINKSTINKS“ zur Unterstützung von Marlis Krämer, nachdem deren Anspruch auf die Bezeichnung „Kundin“ bei Banken vom Bundesverfassungsgericht zurückgewiesen worden war.

Der Verein Deutsche Sprache (VDS) trat 2018 mit dem Aufruf ,Schluss mit dem Gender-Unfug" an die Öffentlichkeit, der im Titel schon stark evaluiert. Hier heißt es, Monika Maron, Wolf Schneider, Walter Krämer und Josef Kraus wendeten sich ,aus Sorge um die zunehmenden, durch das Bestreben nach mehr Geschlechtergerechtigkeit motivierten zerstörerischen Eingriffe in die deutsche Sprache“ mit diesem Aufruf an die Öffentlichkeit. „Die deutsche Sprache" solle einheitlich sein.

Die sehr rechtskonservative Schweizerische Volkspartei antwortet auf die Anfrage des Studenten Fuchs nach ihrer Referenzpraxis:

„Freiheit und Eigenverantwortung gehören zusammen, dafür steht die SVP - auch für Föderalismus und Subsidiarität. So soll es jeder so handhaben bei uns, wie er/sie will. Die SVP Schweiz nimmt fast immer die männliche Form, ausser ${ }^{9}$ bei der direkten Anrede von Frauen und Männern."

(Baer 2015, zitiert nach Fuchs 2015: 54)

Mit Ausnahme der direkten Anrede von offensichtlich gemischtgeschlechtlichen Publika verwendet die Schweizerische Volkspartei das geschlechterübergreifende Maskulinum. Das ist im Kontext der Schweiz, wo öffentlich seit Langem sehr oft gegendert wird, auffällig.

Ähnlich äußert sich auch die Freiheitliche Partei der Schweiz (Fuchs 2015: 58).

Seine Untersuchung eines großen Korpus vieler Textsorten aller Schweizer Parteien zeigt, dass die SVP und die Freiheitlichen zu mehr als 70\% mit generisch gemeintem Maskulinum in ihrem Schrifttum auf Personen referieren. Das bestätigt eine konservative Zuordnung.

Die Registrierung als „konservativ“ ist aber keineswegs die einzig relevante. Einige der Unterstützer/innen des Aufrufs des VDS monieren auch den Gesinnungs- und Bildungsduktus, der dem Reformieren eigen ist, z. B. Feridun Zaimoglu (zitiert nach Orzessek 2019):

„Der Genderjargon markiert. Die Literatur ist aber kein Stadtfaltplan. Die tobenden Frauen und Männer, die sich an der Debatte beteiligen, gehören der bürgerlichen Klasse an. Der Interpunktionsirrsinn ist die Ausgeburt der höheren Töchter. Der Restaurationswahn ist das Gespei der spröden Oberschichtler. Wir sind nicht in der Benimmschule. Ich halte mich nicht an eine Grammatik der Gesinnung.“

(Zaimoglu 2019, zitiert nach Orzessek 2019)

Hier werden geschlechtersensible Schreibstile in gebildeten Kreisen verortet und als Gesinnungsausweis verstanden. Beides ist nicht von der Hand zu weisen (ähnlich argumentiert Biller

\footnotetext{
${ }^{9}$ Schweizerische Orthografie.
} 
2020). Zunehmend sorgt Widerstand gegen den Bildungs- und Richtlinienduktus von Personen, die sich politisch eher links verorten, dafür, dass Typ 1 auch mit dem Widerstand gegen tonangebende Kultureliten assoziiert wird.

In der Presse und in Fachjournalen werden wiederholt textstilistische Bedenken gegen verschiedene Formen des Genderns vorgebracht (cf. Streidl 2017; Zifonun 2018), etwa bezüglich der depersonalisierenden Wirkung neutralisierender Verfahren (z. B. Kollegium) (cf. auch Diewald/Steinhauer 2017) und der Sperrigkeit von mehreren Beidnennungen in einem Satz. Auch werden Bedenken dagegen laut, dass Gender in längeren Texten durch komplexe Personenreferenzen als omnirelevant kommuniziert wird (cf. Bülow/Herz 2015).

\subsection{Das feministische Register}

Neben der Beidnennung (Schwimmerinnen und Schwimmer) wurden zunächst folgende Varianten für eine Kurzschreibung vorgeschlagen: Beidnennung mit Schrägstrich und Beidnennung mit Klammern, später Schreibung des Femininmorphems im Wortinneren mit einem großen I. Pusch vertritt außerdem mitunter ein generisches Femininum [die Studentinnen (Männer sind mitgemeint)] oder generisches Neutrum [das Student] (cf. Pusch 2018). Alle genderfairen Strategien wurden in vielen Richtlinien veranschaulicht (Kargl/Wetschanow/Wodak 1997; Hellinger/Bierbach 1993; Schweizerische Bundeskanzlei 2013). Während generisches Femininum und generisches Neutrum kaum Anwendung fanden und heute eher selten eingesetzt werden (nur in einigen universitären Textsorten, z. B. der Grundordnung der Universität Leipzig), tauchen die anderen genannten Formen im gegenwärtigen Sprachgebrauch oft auf. Die Schreibung mit dem großen I im Wort, die sowohl Femininreferenz symbolisiert als auch durch den Suffigierungsauftakt dann für das Lexem Generizität beansprucht, gehört zentral ins Repertoire. Diese „ehrwürdige feministische Erfindung“ (Pusch in öffentlichen Diskussionen, z. B. in einem Gespräch in rebel-girls 2018) hatte zu vielen Debatten Anlass gegeben; sie wird gelegentlich im akademischen Bereich und in einigen feministischen und verschiedentlich in linken Publikationen (in taz-Artikeln beispielsweise gelegentlich, in der schweizerischen $W O Z^{10}$ sehr häufig) praktiziert. Bei konkreten Personenreferenzen wird das Geschlecht überall fast durchgängig morphologisch ausgedrückt (auch bei Typ 1). Im Bezug auf Angela Merkel ist typenübergreifend von „die Kanzlerin“ die Rede. Generizität vs. Referenzialität lässt sich als Skala abbilden (cf. Tabelle 1).

Nur wenige textlinguistische und textstilistische Abhandlungen beschäftigen sich mit Personenreferenzen, z. B. Petterson (2011) und Kusterle (2011). Kusterle (2011) integriert verschiedene Kotextvariablen, die Genderhinweise verstärken oder abschwächen, in eine Fragebogenstudie, z. B. den Faktor Numerus. Das geschlechterübergreifend gemeinte Maskulinum generiert im Plural etwas ausgewogenere Vorstellungen von Frauen und Männern als der maskuline Singular.

\footnotetext{
${ }^{10}$ In der WOZ-Redaktion wurde er laut Schröter/Linke/Nussbaumer 2012 erfunden.
} 


\begin{tabular}{|c|c|c|c|}
\hline & Beispiele & Kategorien & \multirow{9}{*}{ 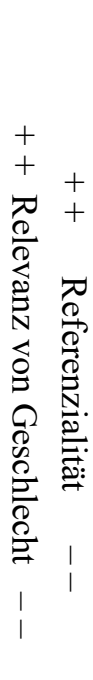 } \\
\hline 1 & Sehr geehrter Rentner! & Adressierend & \\
\hline 2 & Dieser Rentner bezieht gleich sein Zimmer. & $\begin{array}{l}\text { referierend: spezifisch, } \\
\text { demonstrativ, Agens }\end{array}$ & \\
\hline 3 & Der Rentner sucht noch seinen Koffer. & spezifisch, definit, Agens & \\
\hline 4 & Ich habe den Rentner begrüßt. & spezifisch, definit, Patiens & \\
\hline 5 & $\begin{array}{l}\text { Ich begrüße nachher noch einen [bestimmten] } \\
\text { Rentner. }\end{array}$ & spezifisch, indefinit & \\
\hline 6 & $\begin{array}{l}\text { Im Wirtshaus kommt nacher sicher noch [irgend] } \\
\text { ein Rentner vorbei. }\end{array}$ & nicht-spezifisch, indefinit & \\
\hline 7 & $\begin{array}{l}\text { Sg.: Ein Rentner ist immer willkommen; Pl.: Rent- } \\
\text { ner sind immer willkommen. }\end{array}$ & generisch, Subjekt & \\
\hline 8 & Du bist jetzt (ein) Rentner. & Prädikativ & \\
\hline
\end{tabular}

Tabelle 1: Linguistische Kategorien und ihr hypothetischer Bezug zu Referenzialität und Relevanz von Geschlecht (nach Kotthoff/Nübling/Schmidt 2018: 93)

Die Grüne Partei der Schweiz bediente sich der Schreibung mit dem Binnen-I:

Die Grüne Partei ist die Partei der Gleichstellung. [...] Dementsprechend setzen wir uns auch für Gleichstellungsfragen ein, zu denen für uns eine geschlechtergerechte Spracheganz klar dazu gehört. Deshalb sind unsere Texte geschlechtergerecht verfasst, das heisst, sowohl das männliche als auch das weibliche Genus umfassend. Für uns Grüne ist es ein grosses Anliegen, die Frauen sprachlich nicht zu diskriminieren. In gewissen Fällen verwenden wir sogar speziell ausschliesslich das weibliche Genus, wie in unseren Parteistatuten [...]. Zur Einschliessung der weiblichen Form verwenden wir häufig das Suffix -Innen, wie bei MigrantInnen.

(Kamm 2015, zitiert nach Fuchs 2015: 95f.; Hervorhebung i. O.)

Inzwischen ist die Partei auf das nicht-binäre Register umgestiegen (cf. Wahlplattform Grüne Schweiz 2019-2023). Auch die Sozialdemokratische Partei der Schweiz pflegt seit Langem generell in allen Publikationen eine geschlechtergerechte Sprache; das gilt auch für Online-Publikationen (cf. Fuchs 2015: 96). Spezielle Richtlinien dazu gab es zunächst nicht; für sie sei das seit Jahren selbstverständlich. 2017 legte sich die Partei mit Anweisungen auf das nicht-binäre Register fest. Man kann sich bei Festlegungen dieser Art in Zeiten des Ausprobierens neuer Referenzformen durchaus fragen, ob hier nicht ein autoritärer Gestus zu Tage tritt.

\subsection{Das nicht-binäre Register}

In den letzten Jahren ist eine Schreibung mit Unterstrich (Politiker_innen) oder Sternchen/Asterisk (Politiker*innen) hinzugetreten, deren Protagonist(innen) dieses diakritische Zeichen als einen Ausweis von und Hinweis auf den Einbezug von ,transgender“ ansehen und diese Lesart in Diskursen verbreiten (cf. Hornscheidt 2012; Spieß 2013). Auch der DudenRechtschreibung (2020) gibt die Bedeutung des Gendersterns jetzt so an: „(bei Personenbezeichnungen) zwischen Wortstamm beziehungsweise maskuline Flexionsendung und feminine Flexionsendung gesetzter Asterisk, der der sprachlichen Gleichbehandlung aller Geschlechter dienen soll (z. B. Leiter*innen, Pilot*in).“ Laut Hornscheidt (2012) und Baumgartinger (2008) laden beide Grapheme zu einer kognitiven Repräsentation nichtgegenderter Personen ein (dies nenne ich ,queeres Register“). Diese Sprachreform rekon- 
turiert die feministisch inspirierten Vorschläge der neunziger Jahre (Pusch 1984) erheblich und setzt für Typ 2 eine „fraktale Rekursivität“ in Richtung „,von gestern“ und „,binär“ in Gang.

Hornscheidt (2012: 217) lehnt das Femininmorphem-in als „sexistisches mittel“ ab, mit der Begründung, es betreibe „exklusivgenderung personaler appellation für frauisierte über -in-formen und verstärke ,,androgenderung“. Baumgartinger (2008) meint, der Unterstrich eröffne „unendlich viele Begrifflichkeiten zwischen den Geschlechtern.“ Die unterschiedlichen Begründungen für die Semantik dieser Grapheme sind linguistisch kaum zu untermauern (cf. Kotthoff 2017; Kotthoff/Nübling/Schmidt 2018) und zeugen eher von moralischer Aufladung der Texte, die als spezifische Sprachideologie beschreibbar ist und eine erkennbare Gruppenzugehörigkeit indiziert und ikonisiert. Wir finden dieses Register u. a. in Missy, einigen linken und feministischen Internet-Plattformen wie change.org und PINKSTINKS, im Schrifttum der Partei der schweizerischen und deutschen Grünen, manchmal in der Berliner TAZ oder dem wöchentlichen Freitag (Zahlen zu einer Zunahme dieser Schreibungen finden sich in Krome 2020 und in Kolek 2019). Da sich die Zeitschrift Missy häufig von Emma abgrenzt, deutet sich hier eine fraktale Rekursivität an, die wechselseitig eine Konturierung von „feministisch“ und ,postfeministisch“ mit sich bringt.

Der dynamische Unterstrich oder das Sternchen sollen Wörter nach dem Leitfaden der AG Feministisch Sprachhandeln an verschiedenen Stellen unterbrechen; faktisch steht er aber meist vor dem Morphem der Movierung (also Schreiber_innen oder Schreiber*innen). Für beide Grapheme wird eine Palette diverser Verweisfunktionen behauptet. Auf der Web-Seite des Kompetenzzentrums ,gender and diversity“ (TH Nürnberg) heißt es beispielsweise, der Unterstrich

[...] stellt darüber hinaus die Selbstverständlichkeit einer Zwei-Geschlechter-Ordnung und einer heterosexuellen Orientierung als Norm in Frage und will auch denjenigen einen sprachlichen Ort verleihen, die bislang vorwiegend nicht oder nur als „Abweichung“ wahrgenommen werden (Intersexuelle, Transsexuelle, Homo- und Bi-Sexuelle, Transgender, Crossdresser, Drags usw.). Der Unterstrich symbolisiert mithin nicht nur die bisweilen sehr fließenden Übergänge zwischen „Männlichkeit“ und „Weiblichkeit“, sondern auch einen Ort, an dem Überschneidungen und Wanderungen zwischen Geschlechtsidentitäten und sexuellen Orientierungen einen Platz haben.

(Kompetenzzentrum „gender and diversity“ TH Nürnberg 2019)

Es ist bemerkenswert, dass die Verfasser/innen unterstellen, mit maskulinen und femininen Referenzen seien immer Heterosexuelle gemeint. Einem Lexem wie etwa „Schneider“ werden so hinterrücks sexuelle Präferenzen eingeschrieben, die in seiner Semantik aber gar keine Rolle spielen. Solche Behauptungen sind linguistisch und psychologisch fragwürdig und politisch noch mehr. Die durch Referenz flüchtig evozierten Vorstellungen sind vor unserem inneren Auge in all ihrer Flüchtigkeit bildhaft. Woran soll denn die Bisexualität einer Person erkannt werden? Wie könnte dergleichen experimentell getestet werden? Experimentelle Untersuchungen stehen in diesem Bereich noch aus.

Die Vorschläge für einen geschlechtersensiblen Sprachgebrauch der Gleichstellungsbeauftragten der Universität Freiburg (2018) melden über „Verwendung von Unterstrich und Sternchen,“ dies beziehe Personen mit ein, „die sich nicht in das System der Zweigeschlechtlichkeit einordnen.“ „ein_e Forscher_in“ oder „ein*e Stipendiat*in.“Der Leitfaden der Stadt Freiburg der Geschäftsstelle Gender \& Diversity dehnt die Schreibweise sogar auf Referenzen auf die Stadt aus: 
Wir sind sowohl Dienstleister_in für die Bürger_innen wie [...] Ebenfalls handeln wir als zweitgrößte Arbeitgeber_in der Stadt [...]

(Leitfaden der Stadt Freiburg der Geschäftsstelle Gender \& Diversity, zitiert nach Krome 2020: 40)

Die in den Ratgebern getroffene Aussage, der Unterstrich oder das Sternchen stellten die Selbstverständlichkeit der Zwei-Geschlechter-Ordnung in Frage und darüber hinaus auch heterosexuelle Normen, ist linguistisch kaum für jede Personenreferenz begründbar. Ein graphisches Sonderzeichen leistet sicher nicht an jeder Stelle des Auftretens eine solche Gedankenevokation; es ist außerdem nicht transparent, auch nicht als Zeichen für eine spezifische Personenreferenz. Man kann graphische Zeichen durchaus für eine soziale Gemeinschaft mit Appell-Funktionen aufladen, wenn der Metadiskurs darüber intensiv genug betrieben und rezipiert wird. Dann kann ein derartiger Unterstrich für Kreise, die solche Erläuterungstexte rezipieren, einen Wiedererkennungswert symbolisieren. Er kann so zum textuellen Emblem von Gruppenzugehörigkeit werden nach dem Motto: aha, der Text wurde von einer Person verfasst, der viel an der Überwindung der Zweigeschlechtlichkeit liegt. So werden Asterisk und Unterstrich zu Zugehörigkeitszeichen.

Mit den ausdrücklichen Plädoyers für dynamische oder an Morphemgrenzen eingesetzte Unterstriche und Sternchen statt anderer Möglichkeiten der Neutralisierung oder punktuellen Beidnennungen in Personenreferenzen geht es nicht mehr primär um ein Unterlaufen maskulin dominierter Vorstellungen durch die Referenz, sondern auch um eine moralische Aufladung von Texten, die in der ,in-group“ erkannt werden kann, welche sich an entsprechenden Metadiskursen beteiligt. Die von den feministischen Reformer/innen geforderte höhere Präzision, die durch die Beidnennung erreicht werde, ist bei der durch Unterstrich und Sternchen beabsichtigten Dreifachnennung wieder hinfällig, da oftmals auf solche Gruppen so referiert wird, denen keine nichtbinäre Person angehört. So verweist die Plattform campact (z. B. am 27.05.2020) beispielsweise auf Minister*innen, die man aus einem Protestgrund telefonisch anrufen möge und nennt dabei aber nur weibliche und männliche. Die Referenz auf die nichtbinären Minister geht ins Leere. Der Transcript-Verlag schreibt immer von Autor*innen, auch wenn nur eine Autorin und ein Autor genannt werden, die binär sind. Der Asterisk scheint keine Referenzfunktion zu besitzen.

Meiritz (2015) schreibt, dass die deutschen Grünen in ihren Verlautbarungen den Asterisk verwenden:

In der Vergangenheit haben wir auf Landes- und Bundesebene mit verschiedenen Varianten experimentiert", sagt Vorstandsmitglied Gesine Agena, die die Pro-Sternchen-Satzungsänderung federführend entwickelt hat. „Mal wurde das Binnen-I eingesetzt, mal das Sternchen, mal der Unterstrich. Oft kursierten in diesem Zusammenhang zahlreiche Änderungswünsche, die mal die eine, mal die andere Variante bevorzugten. Das kostete Nerven und Papier.

Meiritz (2015)

Mit dem Einsatz des Gender-Stars im Regelfall strebe man nun „ein einheitliches Gendern an“, erklärt die frauenpolitische Sprecherin der Grünen. „Wir halten den Gender-Star für geeignet, weil er das gesamte Spektrum von Geschlechtern und Identitäten berücksichtigt." (zitiert nach Meiritz 2015). Auch hier wird mit „Identitäten“ argumentiert, was über eine Repräsentation nicht-binärer Personen deutlich hinausgeht. Bei jeder Personenreferenz sollen se- 
xuelle Orientierungen mit aufgerufen werden. Eisenberg (2020: 8) verweist auf semiotischikonische Deutungen des Sterns in Richtung „nach allen Seiten offen.“

Schätzle (2019) hat eine erste Online-Einstellungserhebung zur sprachlichen Realisierung von Personenreferenzen durchgeführt. Mit erprobten Methoden hat sie bei etwas über 100 Personen (drei Viertel mit akademischer Bildung, zwei Drittel weiblich, ein Drittel männlich, unterschiedliches Alter) politische Einstellungen, Wissen um Genderdebatten und Einstellungen zu Formen von gegenderter Lang- und Kurzschreibung erhoben. Der erste Abschnitt der Fragebogenstudie bestand aus Fragen zur politischen Einstellung (z. B. „Welche Partei haben Sie zuletzt gewählt?“). Dann sollten die Befragten Aussagen zustimmen, die einem Sexismus-inventar zugeordnet werden. Sie versuchte so, gleichstellungspolitische Einstellungen zu erfassen. Dann gab es einen „Gender-Exposure“. Die Probanden sollten bei 15 Items einen Zusammenhang zur Genderforschung herstellen, darunter fanden sich einige Falschnennungen („False Alarms"). Die Einstellung zu geschlechtergerechter Sprache wurde auf einer Skala von 1-10 über Satzvervollständigungen vorgenommen (notwendig/wichtig vs. unnötig, schön/ästhetisch vs. unschön, genau/präzise vs. verwirrend...). Versuchspersonen, die sich als konservativ einschätzen, bekunden eine kritische Einschätzung zu gendersensibler Sprache. Sehr aufschlussreich ist, wie wenig bekannt die an Universitäten stark propagierten Sonderzeichen Sternchen und Unterstrich insgesamt sind, denen in Leitfäden Referenzfunktionen zugeschrieben werden. In Schätzles Studie (ibd.) zeigt sich, dass selbst von Personen mit akademischem Hintergrund nur die Hälfte den neuen Graphemen Unterstrich und Asterisk eine konkrete Funktion zuordnen kann. Weiterhin zeigt die Studie, dass die hohe Korrelation einer Wertschätzung für Gendern mit beliebigen Zeichen an der Morphemgrenze mit politisch progressiver Einstellung einhergeht und damit assoziiert wird.

$\mathrm{Zu}$ diesem Schreibregister tritt der Buchstabe x, dem von Hornscheidt (2012) eine morphematische Personalisierungsleistung zugeschrieben wird. Fuchs (2015: 9), der sich diesen Hornscheidtschen Setzungen anschließt, fragt:

Wex schliesse ${ }^{11}$ ich aus? Wex benenne ich mit welchen sprachlichen Mitteln?" Hornscheidt betitelt sich entsprechend als „Professx“ um sowohl das Personalisierungsmorphem „or“ als auch das Femininsuffix ,in“ auszuschalten.

Auch Pronomen werden erneuert: Das entsprechende Pronomen zur Wiederaufnahme ist „x“(Wex hat x Mantel vergessen?).

(Fuchs 2015: 9)

Mit weiteren Neuerungen wird Typ 3 angereichert. Hen lautet eines der neuen entgenderten Pronomen, das aus dem Schwedischen übernommen wurde (cf. Das Nichtbinär-Wiki s. v. hen). Die Stadt Lübeck verwendet für ihre offiziellen Verlautbarungen einen Doppelpunkt an der Morphemgrenze (cf. taz vom 01.01.2020).

Der Asterisk wird auch nach Referenznomen wie Mädchen* eingesetzt, beispielsweise in der Zeitschrift betrifft: Mädchen der Landesarbeitsgemeinschaft Mädchen NRW. Prieß et al. (2020: 52) meinen:

11 Schweizerdeutsche Orthografie, da es sich um eine Masterarbeit an der Universität Zürich handelt. 
Der Asterisk ermögliche immer einen Denkraum für die Dekonstruktion der binären Geschlechterkategorien und die Sichtbarmachung von Geschlechtervielfalt. Darüber hinaus verweist das * auch auf die Komplexität der Kategorie ,Mädchen' selbst und ermögliche einen intersektionalen Blick auf Diskriminierungsverhältnisse (...).

(Prieß et al. 2020: 52)

Da aber jede Kategorie komplex ist, könnte man ausgehend von einer solchen Gedankenlinie ganze Texte von oben bis unten mit Asterisken durchsetzen.

Das queere Register wird sprachphilosophisch an einem radikalkonstruktivistischen Denken ausgerichtet, das Sprache als zentrales Mittel der Wirklichkeitsherstellung ansieht (Petterson 2011: 70). Entsprechend sieht Hornscheidt (2006) Referenz als Akt der Herstellung und verwendet deshalb den Terminus „Appellation“. Wenn ich jemanden anrufe, mache ich ihn/sie zum Angerufenen. Der Terminus geht über in der Linguistik gängige Vorstellungen von Referenz hinaus, die aus Sicht Hornscheidts ,die Auffassung einer objekthaften Vorgängigkeit der Welt vor der sprachlichen Benennung [tradiert und reproduziert]." (Hornscheidt 2006: 52). Wenn wir etwa auf Bäume oder Kinder referieren, waren diese bereits in einer (möglichen) Welt vorhanden. Weder Typ 1 noch Typ 2 sind vergleichbar idealistisch-konstruktivistisch konzipiert. Zumindest werden die Grenzen des sprachlich Herstellbaren bei Hornscheidt nicht deutlich. Eine Lehrerin wird nicht zu einer solchen durch den Referenzakt, sondern war es durchaus auch vorher schon, wenn sie professionell gelehrt hat und in entsprechenden Kontexten die Rolle spielt, die die Referenz nachvollziehbar macht.

Wenn die Sprachmacht einerseits als so umfassend angesehen wird, andrerseits die Funktion der Grapheme nicht deutlich ist, tritt die moralische Dimension des Registers sehr in den Vordergrund. Das zeigt sich auch bei Diskussionsversuchen über das Register.

Es gibt inzwischen kritische Berichte von Personen, die heutige Praktiken des Genderns in ihrem Für und Wider beleuchten möchten und sich bei nachdenklichen Äußerungen sofort angegriffen sehen; so berichtet es z. B. die Philosophin Svenja Flaßpöhler im Gespräch mit Harald Welzer und Peter Unfried bei FUTURZWEI (2019):

Svenja Flaßpöhler: Im juristischen Sinne natürlich niemand, aber im moralischen Sinne würden mir manche Leute gerne den Mund verbieten. Kleines Beispiel: Ich habe vor einigen Wochen bei einer Debatte im Literarischen Colloquium Berlin versucht, meine Haltung zur gendergerechten Sprache darzulegen, die ambivalent ist. Ich habe versucht, einen Denkraum zu eröffnen und das Publikum zum offenen Austausch einzuladen. Unter anderem war Lann Hornscheidt da, begleitet von zwei exakten Kopien seiner selbst, gleiches Hemd, gleiche Frisur, beide sahen genauso aus wie er, hat mich schon etwas belustigt, diese Uniformierung...

tazFUTURZWEI: Hornscheidt ist laut Website „Profess_x für Gender Studies und Sprachanalyse“. Spezialgebiet: geschlechtsneutrale Sprache. Warum sagen Sie „der Lann Hornscheidt“?

Svenja Flaßpöhler: Hätte ich „das Lann Hornscheidt“ sagen sollen? Hier zeigt sich, dass Sprache widerspenstig ist, dass sie eine historisch gewachsene, grammatikalisch-semantische Eigenlogik hat und sich nicht dem emanzipatorischen Willen einzelner unterwirft: „Das“ klingt eben extrem verdinglichend, und ich will niemanden verdinglichen. Ich hätte natürlich auch „die Lann Hornscheidt"sagen können. Dann hätte ich mich am biologischen Geschlecht orientiert. Als Frau, die durch die poststrukturalistische Theoriebildung gegangen ist, habe ich mich ganz subversiv für „der“ entschieden. Aber zurück zu meiner kleinen Geschichte: Im LCB damals waren auch 
junge Feministinnen. Die haben überhaupt nicht ausgehalten, dass da jemand auf der Bühne auch die kritische Seite der gendergerechten Sprache beleuchtet. Also etwa die Frage stellt, wie offen Sprache für die Forderungen einzelner ist, so oder so angeredet zu werden. Die aggressiven Zwischenrufe kulminierten dann in dem Satz: „Hören Sie endlich auf, Sie beleidigen uns!“

Eigene Gesprächserfahrungen mit diesem Paradigma Nahestehenden deuten auch in die Richtung eines schnellen Beleidigtseins und dezidierten Desinteresses, wenn ich problematisiere, dass einige der Begründungen für die Unterstrich- und Sternchenschreibung dubios und inkonsistent sind. Das zieht Ausladungen von Vorträgen nach sich.

Aber auch wenn man nur die These verfolgte, es würde mit den neuen Graphemen auf nichtbinäre Personen verwiesen, sollte das nachdenklich stimmen, weil es von denen, die sich so auch stilisieren, nur etwa 20000 Personen in Deutschland gibt (Spiewag 2019). Die große Mehrheit der Transsexuellen ordnet sich weiblich oder männlich zu - nicht divers. Eine laufende Repräsentanz einer solch kleinen Gruppe beim Lesen zu leisten, ist eher unwahrscheinlich. Kognitive Repräsentation funktioniert nicht nach abstrakten Moralkategorien, sondern benötigt zunächst eine gewisse Frequenz der Personentypen, auf die referiert wird, im alltäglichen Erfahrungsbereich. Wenn ich viele nicht-binäre Schneider*innen kenne, werde ich mir diese beim Lesen oder Hören auch so vorstellen, wie ich sie eben kenne, egal, welches Graphem im Wort steckt. Aber weder bezüglich des Lesens noch des Hörens gibt es bis dato Studien, die etwa für „Leser_innen“ mit dem Glottisschlag und dem dadurch separierten „innen“ nachweisen würden, dass in der Schnelle des Leseprozesses drei Personentypen vor das innere Auge treten.

Pusch (2019) kritisiert das Asterisk auf die Frage im On-line-Journal jetzt so:

Transgender oder Intersexuelle sind bis zum Gendergap oder Gendersternchen grammatikalisch gar nicht aufgetaucht. Ist es nicht noch schlimmer, wenn eine Gruppe sprachlich unsichtbar ist, als wenn sie sprachlich untergeordnet ist?

Viele Gruppen wurden oder werden grammatikalisch nicht gekennzeichnet, ihre Existenz wird aber im Wortschatz ausgedrückt, ob das nun Schwarze sind oder Menschen mit Behinderung. Für sie alle gibt es keine grammatischen Endungen - die gibt es nur für Frauen, das ,-in“, was an sich schon diskriminierend ist. Es ist aber auch ein Argument der Menge: Die sprachliche Diskriminierung von Frauen betrifft 52 Prozent der Bevölkerung, die Transgender-Community macht weit weniger als ein Prozent aus. Natürlich hat sie auch sprachliche Rechte, und es ist richtig, dass sie in die Debatte um Männer und Frauen eingreift, weil es auch bei ihr um das Geschlecht geht aber das Gendersternchen ist nicht die richtige Lösung.

Pusch (2019)

Zifonun (2018: 52) problematisiert, dass auch Vorschläge der Aussprache der Sonderzeichen mit Glottisschlag, wie sie sich im „Sprachlog“ von Stefanowitsch (2018) finden, erstens nur in eingeweihten Kreisen nachvollzogen würden und zweitens würde die männliche Referenz dadurch stärker hervorgehoben, da das mit Glottisschlag abgesetzte neue Suffix (z. B. *in) nun auch nur noch eine Referenz auf nichtmännliche Personen evoziere (ergo männlich vs. nichtmännlich). Die Aussprache ist im Deutschen silbisch organisiert und nicht morphematisch (Tän-zer-rinnen und nicht Tänz-er-innen), wie es diese Richtung jetzt für die mit Glottisschlag abgetrennten Femininmorpheme vorschlägt. Ich bin Au-to-rin dieses Artikels (so ist die Silbenstruktur), nicht Au-tor-in. Da die Morpheme innen auch in Komposita eingesetzt werden (z. B. seit einigen Wochen von der Fernsehmoderatorin Anne Will in der sonntäglichen Talk- 
runde auf ARD und auch im Deutschlandfunk), entstehen solche Wortgebilde Bürger-innensaal. Ist das Gegenstück nicht der Bürgeraußensaal? Die Glottisschläge dürften sich nicht von selbst verstehen. Eisenberg (2020: 9) schreibt, dass wortprosodische Grundregularitäten des Deutschen diese Gruppe nicht kümmern.

Der Philosoph Robert Pfaller beklagt in einem Interview mit TAZ und FUTURZWEI (Nr. 9, August 2019), der starke Fokus auf dem „korrekten Sprechen“ linkskultureller Kreise lenke von handfester Politik ab. Sowohl das Binnen-I als auch der Gender-Stern seien Erkennungszeichen, somit identitäre Zeichen, die in den Dienst von Distinktion gestellt würden. Das schraube persönliche Empfindlichkeiten und Moral hoch, statt nach gruppenübergreifenden Gemeinsamkeiten im Kampf gegen den globalen Kapitalismus Ausschau zu halten. Dieses Hochschrauben persönlicher Empfindlichkeit hängt mit der oben kritisierten enormen Machtzuschreibung an Sprache zusammen. Eine ähnlich gelagerte Kritik an identitätspolitischen Bestrebungen im linken Spektrum macht auch Biller (2020: 48) u. a. am Gender-Stern fest.

\subsection{Vielfältiges Gendern}

Bei verschiedenen Radiosendern, z. B. dem Ö1 (dazu Schrodt 2018) und SRF 2, finden sich oft Einführungen von Personengruppen mit Beidnennung. Im Laufe des Beitrags tauchen diese mehr oder weniger oft wieder auf, meist dominiert nur phasenweise ein übergreifend gemeintes Maskulinum im Plural. Bei beiden Sendern sind Formen von „,punktuellem Gendern“ (Typ 4) mit einer allmählichen Zunahme über die letzten Jahre hinweg heute an der Tagesordnung, wie meine eigene tägliche Hörerfahrung zeigt.

Allgemein bekannt, aber im Bezug auf Gendereffekte kaum untersucht, ist aber auch, dass in Pressetexten bei spezifischer personaler Referenz Bezeichnungen wechseln (z. B. Name, Berufsbezeichnung, Funktionsbezeichnung, Pronomen) und so Wiederholungen vermeiden und gleichzeitig Informationsdichte bis hin zu Bewertung herstellen (z. B. Alice Schwarzer, die Emma-Herausgeberin, die Journalistin, die kämpferische Feministin usw.). Pettersson (2011: 92) macht in vielen Zeitschriften (darunter auch Emma) die wichtige Feststellung, dass ,überwiegend Maskulina zur nicht-spezifischen und Beidbenennungen zur spezifischen geschlechtsübergreifenden Referenz benutzt werden“. Damit strukturiert und steuert Referenzialität die Wahl geschlechtsübergreifender Personenbezeichnungen: Je eher Personen konkretisiert oder gar identifiziert werden, desto eher Beidnennung, je eher sie diffus im Hintergrund verbleiben, desto eher geschlechterübergreifendes Maskulinum und Neutralformen. Erwartbarerweise sind in Emma geschlechterübergreifende Maskulina eher selten, und wenn sie vorkommen, dann in nicht-spezifischer Referenz (nicht-referenziell) und v. a. im „entschärften“ Plural, z. B. Studenten, Ausländer, Berufsberater sowie prädikativ Teenager, die allesamt für das Textthema irrelevant sind. Die über explizite Formen (Beidnennungen, großes I) bezeichneten Personen sind konkretisiert sowie spezifiziert, und vor allem sind sie für das Textthema zentral („Relevanzprinzip“). Damit unterscheiden Personenreferenzen auch zwischen thematisch wichtig und unwichtig, zwischen zentralen und peripheren Personen, zwischen Nähe und Distanz.

In wissenschaftlichen Büchern, z. B. bei dem Soziologen Knoblauch (2017) und der Germanistin Kopf (2014) finden sich neue Schreibpraktiken des in Kapiteln wechselnden Einsatzes eines generisch gemeinten Maskulinums und eines generisch gemeinten Femininums. Vor allem bei 
Büchern ist dies, wenn man außerdem in einer Fußnote erklärt, was man tut und warum, eine Möglichkeit, die zumindest im Unterschied zu Typ 3 ohne Leitfaden auskommt.

Die Effekte solcher Formen des nicht durchgängigen Genderns wurden bis dato nicht erforscht; es ist zu vermuten, dass sie durchaus geeignet sind, für eine geschlechterausgewogene Repräsentation zu sorgen und ein nichtakademisches Publikum an solche Stile zu gewöhnen.

Einige Parteien schreiben ihre Verlautbarungen in einem Stil des Typ 4, z. B. die schweizerische CVP, die insbesondere Doppelformen und ,neutrale“ Benennungen verwendet. Generische Maskulina werden hier nicht kategorisch ausgeschlossen (cf. Fuchs 2015: 48).

Auch die FDP der Schweiz führt keine offiziellen Vorschriften oder Richtlinien über einen geschlechtergerechten Sprachgebrauch. Sie halten dies insofern auch nicht für notwendig, als dass in der heutigen Zeit ein gewisses Bewusstsein für geschlechtergerechtes Schreiben durchaus gebräuchlich sei:

Wir sind jedoch gegen eine strikte Anwendung von Richtlinien, wie der Bund es pflegt. Die Aufführung beider Formen kann die Leserlichkeit eines Textes stark beeinflussen. Daher werden nicht konsequent beide Formen aufgeführt. Wo immer möglich werden Paarbezeichnungen wie „Mitarbeitende“ verwendet.

(Darendinger 2015, zitiert nach Fuchs 2015, 69)

\section{Schluss: Metapragmatische Positionierungen}

Metapragmatik ist ein Begriff aus der semiotisch informierten linguistischen Anthropologie, der auf Silverstein (1996) zurückgeht und beschreibt, wie die Auswirkungen und Bedingungen der Sprache selbst zu Objekten des Diskurses werden. Dazu gehören auch mit dem Sprachstil bewirkte kontextuelle und soziale Zuordnungen, die als Registrierungen gefasst werden (Agha 2003; Droste 2017). Sobald ein Merkmal salient ist, kann ihm im Kontext soziale Bedeutung zugeschrieben werden, die es als Kontextualisierungsverfahren mit sich trägt.

Obwohl noch keine quantitativ-variationistische Studie zu den personenreferentiellen Stilen vorliegt, kann man durchaus Angaben zu typischen Zuordnungen vornehmen, wie ich es im Laufe des Artikels getan habe. Es wundert nicht, dass der Schreibstil Typ 1 sich in sprachkonservativen Medien findet (in FAZ und NZZ publizieren Glück (2018), Eisenberg (2017) und Bayer (2019) auch ihre Sichtweise) und deren sprachbezogene Konservativität mitkreiert. Die politisch rechts orientierte deutsche AfD und die schweizerische SVP lehnen Gendern ab. Beidnennung ist allerdings bei Ansprachen und konkreten Referenzen überall selbstverständlich. Durch kritische Stellungnahmen von Autor/innen zum Gendern stellen sich zunehmend auch Assoziationen zu hohen textästhetischen Anforderungen her und zur Ablehnung von möglichen Vorschriften.

Nachdem sich im deutschsprachigen Raum personenreferentielle Stile diversifiziert haben, wird auch das früher einzig Übliche markant und übernimmt eine Index-Funktion, indem es mit Kontexten seines Auftretens assoziiert wird (Kotthoff 2012). Die sprachkonservative Haltung verwechseln wir nicht mit einer Vorliebe für patriarchale Strukturen. Alle AutorInnen betonen ihr Interesse an einer Gleichberechtigung der Geschlechter, aber sie sehen Morphologie nicht als Faktor der Emanzipation. Sie gilt ihnen nicht als bewusstseinsprägend. AutorInnen, die die diese Position einnehmen, wie Eva Menasse und Feridun Zaimoglu in der „Zeit“ weisen vor 
allem den Bürokratieduktus der gendergerechten Sprache von sich, nicht aber Gleichberechtigungsansprüche. Ähnlich äußert sich Biller (2020).

Der Schreibstil Typ 2 entstand im Kontext feministischer Anliegen und wurde von Vertreter/innen des Typ 3 metakommunikativ als „binär“ rekonturiert und damit gleichzeitig als gendertheoretisch veraltet (dazu Wetschanow 2017). In der Zeitschrift Emma und der WOZ wird er weiterhin fast durchgängig praktiziert, in der TAZ, im Freitag oder im Falter ist er auch noch vertreten.

Typ 3 soll Unterschiedliches leisten, vom Einbezug diverser Personen über den von Homosexuellen bis zum dauernden Reflektieren von Gender, das beim Lesen zu absolvieren sei. Bis dato gibt es nur eine Studie, deren Ergebnisse zeigen, dass bei Lektüre solcher Texte nichts dergleichen stattfindet. Weitere Studien müssen wir abwarten. Auf jeden Fall beziehen sich Typ 2 und 3 fraktal rekursiv aufeinander und kennzeichnen so Distinktionen innerhalb der um Geschlechtergerechtigkeit bemühten Kreise und Praktiken. Einer politischen Solidarität dient das nicht. Wir berühren also den Zusammenhang von (Sprach)Politik und Moral. Typ 2 und Typ 3 treten mit dezidiertem moralischem Anspruch auf. Bei Typ 3 überschreibt der moralische Anspruch die Klarheit der beabsichtigten Effekte und kümmert sich auch nicht um den diesbezüglichen Forschungsstand.

$\mathrm{Zu}$ Typ 4 finden sich keine eigenen Metadiskurse. Er wird einfach praktiziert (wenngleich er im Hintergrund natürlich von den anderen Diskursen ums Gendern profitiert). Auf der Graphemebene kann hier der Schrägstrich dazu gezählt werden, dessen Semantik so transparent ist, dass sie keiner Erläuterungen bedarf. Im Bereich der Stellenanzeigen jeder Couleur ist er ein häufig verwendetes Zeichen. Schrodt (2018) zeigt für viele Beiträge des Radios Ö1, dass sich bei Referenzen auf Individuen ein Muster herausbildet, in dem mit einem geschlechterübergreifenden Maskulinum auf Rollenträger referiert wird und die Beidnennung die so Bezeichneten stärker ins Empathiezentrum rückt (ähnlich Petterson 2011).

Dem Stil des punktuellen Genderns ist im Unterschied zu allen drei anderen Stilen kaum missionarischer Eifer eigen. Silverstein (1996) reserviert ja die Attribution von Sprachideologie für metalinguistische Aussagen, die auf sozial-kognitive Funktionen von Legitimierung eines Sprachgebrauchs abheben und Dominierung anstreben. Dies trifft nur auf Typ 4 nicht zu. Er scheint mit Varianz gut zurechtzukommen, für ihn werden keine Leitlinien entworfen und er kämpft auch nicht metadiskursiv gegen andere Stile an.

In diesem Artikel wurden anthropologisch-linguistische Konzepte von Sprachideologie auf die gegenwärtige Debatte rund ums Gendern im Deutschen bezogen. Diese Konzepte fordern in Zukunft durchaus mehr Auseinandersetzung, als im vorliegenden Artikel geleistet werden konnte.

\section{Literaturverzeichnis}

Agha, Asif (2003): "The social life of a cultural value". Language and communication 23: 231-73.

Agha, Asif (2007): Language and social relations. Cambridge: CUP.

Androutsopoulos, Jannis (2006): „Ethnolekte in der Mediengesellschaft. Stilisierung und Sprachideologie in Performance, Fiktion und Metasprachdiskurs“. In: Fandrych, Christian/ 
Salverda, Reinier (eds.): Standard, Variation und Sprachwandel in germanischen Sprachen. Tübingen, Narr: 113-155.

Baumgartinger, Perrson P. (2008): „Lieb[schtean] Les[schtean], [schtean]. Von Emanzipation und Pathologisierung, Ermächtigung und Sprachveränderungen“. Liminalis 02: 24-39.

Bayer, Josef (2019): „Sprachen wandeln sich immer, aber nie in Richtung Unsinn.“ Neue Zürcher Zeitung [10.04.2019].

Biller, Maxim (2020): “Das eiskalte Aufklärungsmanifest”. Die ZEIT 24: 48-49.

Blommaert, Jan (2005): Discourse. Cambridge: Cambridge University Press.

Bourdieu, Pierre (1983) : «Le changement linguistique : entretien avec William Labov ». Actes de la Recherche et Sciences Sociales 46/67-71.

Braun, Friederike et al. (1998): „Können Frauen Geophysiker sein? Generische Personenbezeichnungen im Deutschen." ZGL 26: 265-283.

Bülow, Lars/Herz, Matthias (2015): „Undoing Gender? Ein Abgleich sprachpolitischer Maßnahmen in Rechtstexten mit dem tatsächlichen Sprachgebrauch junger Frauen“. Muttersprache 2/2015: 133-155.

Busch, Florian (2018): Digitale Schreibregister von Jugendlichen analysieren. In: Ziegler, Arne (ed.): Jugendsprachen. Aktuelle Perspektiven internationaler Forschung. Berlin/Boston, de Gruyter: 829-857.

Busch, Florian/Droste, Pepe/Wessels, Elisa (2019): ,, Reflexivität kommunikativer Praktiken. Neue Perspektiven auf Metakommunikation.“ Einladungstext zur Tagung 29.-30.08.2019 an der Universität Hamburg. slm.uni-hamburg.de/germanistik/forschung/veranstaltungen/ reflexivitaet-2019.html [17.07.2020].

Campact: campact.de [17.07.2020].

Das Nichtbinär-Wiki. nibi.space/pronomen\#hen [14.07.2020].

Diewald, Gabriele/Steinhauer, Anja (2017): Richtig gendern: Wie Sie angemessen und verständlich schreiben. Berlin: Duden Verlag.

Droste, Pepe (2014): „Rezension zu Asif Agha ,Language and Social Relations““. Gesprächsforschung. Online-Zeitschrift zur verbalen Interaktion 15: 109-114.

Droste, Pepe (2017): „,Enregisterment'aus praxisorientierter Perspektive - Der Fall narrativ inszenierter Kontrastierung von Varietätengebrauch". Arbeitspapierreihe Sprache und Interaktion 78/12. arbeitspapiere.sprache-interaktion.de [10.07.2020].

Duden-Grammatik (1998): Duden. Grammatik der deutschen Gegenwartssprache. 6., neu bearbeitete Auflage. Mannheim etc.: Dudenverlag.

Duden-Grammatik (2016): Duden. Die Grammatik: Unentbehrlich für richtiges Deutsch. 9., vollständig überarbeitete und aktualisierte Auflage. Berlin: Dudenverlag.

Duden-Rechtschreibung (2020): duden.de/rechtschreibung/Genderstern [05.09.2020].

Eisenberg, Peter (2017): „Das missbrauchte Geschlecht“. Süddeutsche Zeitung [02.03.2017].

Eisenberg, Peter (2020): Die Vermeidung sprachlicher Diskriminierung im Deutschen. Muttersprache. Vierteljahresschrift für deutsche Sprache 130, März, 3-17.

Ferstl, Evelyn C./Kaiser, Anelis (2013): „Sprache und Geschlecht: Wie quantitative Methoden aus der Experimental- und Neuropsychologie einen Beitrag zur Geschlechterforschung leisten können“. GENDER: Zeitschrift für Geschlecht, Kultur und Gesellschaft 5/3: 9-25.

Flaßpöhler, Svenja (2019): „Die Philosophin S. F. über militante Intoleranz. Gespräch mit Harald Welzer und Peter Unfried“". tazFUTURZWEI 9: 14-24. 
Fridays for Future. Deutsche Webseite: fridaysforfuture.de [10.07.2020].

Fuchs, Silvio (2015): Die Schweizer Demokratxs? Politische Parteien der Schweiz und gendergerechte Personenreferenz. Masterarbeit, Universität Zürich. ds.uzh.ch/_files/ uploads/studarb/44.pdf [14.07.2020].

FUTURZWEI Nr. 9 2019: futurzwei.org/article/1192 [02.06.2020].

Gauger, Hans-Martin (2017): „Herr Professorin?“ In: Meinunger, André/Baumann, Antje (eds.): Die Teufelin steckt im Detail. Zur Debatte um Gender und Sprache. Berlin, Kadmos: $67-71$.

Gleichstellungsbeauftragte der Universität Freiburg (2018): Vorschläge für einen geschlechtersensiblen Sprachgebrauch. gleichstellungsbuero.uni-freiburg.de/de/download /Uni_FlyerGBLeitlinie15_E1_DL_6Seiter_Print_CMYK_CS52.pdf [14.07.2020].

Gleichstellungsbeauftragte der Universität zu Köln (2017): „ÜberzeuGENDERe Sprache. Leitfaden für eine geschlechtersensible und inklusive Sprache“. gedim.unikoeln.de/sites/genderqm/user_upload/Leitfaden_geschlechtersensible_Sprache_5.Auflage_ 2017.pdf [14.07.2020].

Glück, Helmut (2018): „Eine kleine Sex-Grammatik.“ FAZ [02.05.2018].

Gramsci, Antonio (1991): Gefängnishefte. Hrsg. von Klaus Bochmann und Wolfgang Fritz Haug. 10 Bände. Hamburg: Argument.

Grundordnung der Universität Leipzig: stura.uni-leipzig.de/doc/debatte-um-generischesfemininum-der-grundordnung-der-universitaet-leipzig-beweist-strukturelle [01.02.2020].

Gumperz, John (1992): Contextualization and Understanding. In: Alessando Duranti and Charles Goodwin (eds.): Rethinking Context. Cambridge, Cambridge University Press: 229-252.

Gygax, Pascale et al. (2008): "Generically intended, but specifically interpreted: When beauticians, musicians, and mechanics are all men". Language and Cognitive Processes 23: $464-485$.

Haspelmath, Martin (2006): „Against markedness (and what to replace it with).” Journal of Linguistics 42/1: 25-70.

Haspelmath, Martin (2019): „Ist die Gender-Grammatik biologisch vorbestimmt? Eine Antwort auf Josef Bayer". dlc.hypotheses.org/1730 [03.06.2020].

Hellinger, Marlis/Bierbach, Christine (1993): Eine Sprache für beide Geschlechter. Bonn: Deutsche Unesco-Kommission.

Hornscheidt, Lann (2006): Die sprachliche Benennung von Personen aus konstruktivistischer Sicht. Genderspezifizierug und ihre diskursive Verhandlung im heutigen Schwedisch. Berlin/New York: de Gruyter.

Hornscheidt, Lann (2012): Feministische w_orte. Frankfurt: Brandes \& Apsel.

Irvine, Judith (1989): "When talk isn't cheap: language and political economy". American Ethnologist 16/2: 248-67.

Irvine, Judith/Gal, Susan (2000): "Language Ideology and Linguistic Differentiation". In: Kroskrity, Paul (ed.): Regimes of Language. Ideologies, Polities, and Identities. Santa Fe, School of American Research Press: 35-83.

Kargl, Maria/Wetschanow, Karin/Wodak, Ruth (1997): Kreatives Formulieren. Anleitungen zu geschlechtergerechtem Sprachgebrauch. Wien: Schriftenreihe des Frauenministeriums. Band 13. 
Knoblauch, Hubert (2017): Die kommunikative Konstruktion der Wirklichkeit. Wiesbaden: Springer.

Kolek, Vit (2019): „Discourse of Non-Heteronormative Labelling in German-Language Press: The Case of Gendersternchen“. Slovenscina 2.0 7/2: 118-140.

Kompetenzzentrums Gender \& Diversity, TH Nürnberg: th-nuernberg.de/einrichtungengesamt/kompetenzzentren/kompetenzzentrum-gender-diversity/kompetenzzentrum-genderdiversity/ [06.08.2019].

Kopf, Kristin (2014): Das kleine Etymologicum. Eine Entdeckungsreise durch die deutsche Sprache. Stuttgart: Klett-Cotta.

Kotthoff, Helga (2012): „Indexing gender““ unter weiblichen Jugendlichen in privaten Telefongesprächen“. In: Günthner, Susanne et al. (eds.): Genderlinguistik. Berlin/New York, de Gruyter: 251-287.

Kotthoff, Helga (2017): „Von Syrx, Sternchen, großem I und bedeutungsschweren Strichen. Über geschlechtergerechte Personenbezeichnungen in Texten und die Kreation eines schrägen Registers“. Osnabrücker Beiträge zur Sprachtheorie 90: 91-117.

Kotthoff, Helga/Nübling, Damaris/Schmidt, Claudia (2018): Genderlinguistik. Eine Einführung in Sprache, Gespräch und Geschlecht. Tübingen: Narr.

Krome, Sabine (2020): “Zwischen gesellschaftlichem Diskurs und Rechtschreibnormierung". Der Sprachdienst 1/2, 35-46.

Kusterle, Karin (2011): Die Macht von Sprachformen. Der Zusammenhang von Sprache, Denken und Genderwahrnehmung. Frankfurt: Brandes \& Apsel.

Landesarbeitsgemeinschaft Mädchen*arbeit NRW: Betrifft Mädchen. maedchenarbeitnrw.de/lag/betrifft-maedchen.html [14.07.2020].

Lehmann, Anna (2019): „Linke will auf Gendern verzichten“. Taz 03.09.2019. taz.de/Gendern/!t5580489 [14.07.2020].

Leitfaden der AG Feministisch Sprachhandeln: feministisch-sprachhandeln.org/wpcontent/uploads/2014/03/onlineversion_sprachleitfaden_hu-berlin_2014_ag-feministischsprachhandeln.pdf [10.02.2018].

Lewandowski, Theodor (1994): Linguistisches Wörterbuch Bd. 1. 4. Aufl. Heidelberg: UTB.

Lobin, Henning/Nübling, Damaris (2018): „Genus, Sexus, Nexus“. Süddeutsche Zeitung [07.06.2018].

Meiritz, Annett (2015): „Grüne wollen den Gender-Star* ganz groß rausbringen“. Spiegel online. 18.11.2015. spiegel.de/politik/deutschland/die-gruenen-machen-geschlechtsneutralesprache-zur-pflicht-a-1063228.html [09.07.2020].

Orzessek, Arno (2019): „Streit ums Gendern von 'Pflicht' bis 'Quatsch'“. deutschlandfunkkul tur.de/aus-den-feuilletons-streit-ums-gendern-von-pflicht-bis.1059.de.html?dram:article_id $=419733$ [02.06.2020].

Petterson, Magnus Ängsal (2011): Geschlechtsübergreifende Personenbezeichnungen. Eine Referenz- und Relevanzanalyse an Texten. Tübingen: Narr.

Pfaller, Robert (2019): Der Schriftsteller R. P. über Gendersternchen als neoliberales Distinktionsinstrument von Kulturlinken. taz/FUTURZWEI 9: 44-53.

PINKSTINKS: pinkstinks.de/vielen-dank-fuer-deine-unterschrift/ [10.07.2020].

Prieß, Jeannine et al. (2020): „Das * in der feministischen Mädchen*arbeit - mehr als gendersensible Sprache“. Betrifft Mädchen 33/2: 52-56. 
Pusch, Luise (1979): „Der Mensch ist ein Gewohnheitstier, doch weiter kommt man ohne ihr. Eine Antwort auf Kalverkämpers Kritik an Trömel-Plötz Artikel über ,Linguistik und Frauensprache“". Linguistische Berichte 63: 84-102.

Pusch, Luise (1984): Das Deutsche als Männersprache. Frankfurt: Suhrkamp.

Pusch, Luise (2018):,,Fühlen Sie sich mitgemeint“. emma.de/artikel/fuehlen-sie-sichmitgemeint-336301 [10.07.2020].

Pusch, Luise (2019): „Das Gender-Sternchen ist nicht die Lösung“. jetzt.de/hauptsachegendern/gendern-linguistin-luise-f-pusch-ueber-das-gendersternchen-und-geschlechterge rechte-sprache [10.07.2020].

rebel-girls (2018): „Von Knacklauten und Sternchen, Gendersensibler Sprache - Sookee im Gespräch mit Luise Pusch“. rebel-girls.de/von-knacklauten-und-sternchen-gendersensiblesprache-socke-im-gespraech-mit-luise-pusch [15.07.2020].

Schätzle, Dara (2019): Metadiskurse zu gendersensibler Sprache. Kognitivlinguistische, grammatische und textstilistische Argumente. Masterarbeit in der Germanistischen Linguistik der Universität Freiburg. Publiziert als Freiburger Arbeitspapier zur germanistischen Linguistik 40. portal.uni-freiburg.de/sdd/fragl/Frag1\%2040/files/frag140.pdf [14.07.2020].

Schrodt, Richard (2018): „Genus, Sexus, Sprache und Schreibung: Gendern“. LiThes. Zeitschrift für Literatur und Theatersoziologie 11/15: 20-39. lithes.uni-graz.at/lithes/beitraege $18 \_15 /$ schrodt_genus_sexus_gendern.pdf [14.07.2020].

Schröter, Juliane/Linke, Angelika/ Bubenhofer, Noah (2012): „, Ich als Linguist ${ }^{\star}$-Eine empirische Studie zur Einschätzung und Verwendung des generischen Maskulinums“. In: Günthner, Susanne et al. (eds.): Genderlinguistik. Sprachliche Konstruktionen von Geschlechtsidentität. Berlin/Boston, de Gruyter: 359-379.

Schweizerische Bundeskanzlei (2013): Geschlechtergerechte Sprache. Leitfaden zur geschlechtergerechten Formulierung im Deutschen. 3. Aufl. Bern: Bundeskanzlei.

Silverstein, Michael (1996): "Shifters, Linguistic Categories, and Cultural Description". In: Basso, Keith/Selby, Henry (eds.): Meaning in Anthropology. Albuquerque: University of New Mexico Press: 11-55.

Silverstein, Michael (2003): Indexical order and the dialectics of sociolinguistic life. Language\&Communication 23: 193-229.

Spieß, Constanze (2013): „Binnen-I, Beidnennung, Neutralform oder Unterstrich? “Der Deutschunterricht1: 75-79.

Spiewag, Martin (2019): „Diverse Missverständnisse“. Die Zeit 20/2019. zeit.de/2019/20/drittes-geschlecht-intersexualitaet-gender-gleichberechtigung-personenst andsrecht [10.06.2020].

Spitzmüller, Jürgen (2005): Metasprachdiskurse. Einstellungen zu Anglizismen und ihre wissenschaftliche Rezeption. Berlin/New York: de Gruyter.

Spitzmüller, Jürgen (2015): "Graphic variation and graphic ideologies. A metapragmatic approach”. Social Semiotics 25/2: 126-141.

Stahlberg, Dagmar/Sczesny, Sabine (2001): „Effekte des generischen Maskulinums und alternativer Sprachformen auf den gedanklichen Einbezug von Frauen“. Psychologische Rundschau 52/3: 131-140. 
Stefanowitsch, Anatol (2018): „Gendergap und Gendersternchen in der gesprochenen Sprache“. sprachlog.de/2018/06/09/gendergap-und-gendersternchen-in-der-gesprochenen -sprache [27.12.2018].

Streidl, Barbara (2017): „Das böse Ende. Linguistik: Ob Sternchen helfen?“ Der Freitag 44/2017. digital.freitag.de/4417/das-boese-ende [16.08.2019].

taz, Interview zum Thema „Inklusive Sprache in den Medien: Intuition und Abwehr“: taz.de//Inklusive-Sprache-in-Medien/!5688436 [14.07.2020].

taz vom 07.06.2020: taz.de/Hansestadt-schreibt-genderneutral/!5653215 [14.07.2020].

Van Dijk, Teun (1995): "Discourse analysis as ideological analysis". In Schäffner, Christina/Wenden, Anita (eds.): Language and Peace. Aldershot. Dartmouth: 17-33.

Verein Deutsche Sprache (VDS): vds-ev.de/gegenwartsdeutsch/gendersprache/gendersprache -unterschriften/schluss-mit-dem-gender-unfug [10.07.2020].

Verwaltung der Stadt Hannover: hannover.de/Leben-in-der-Region-Hannover/VerwaltungenKommunen/Die-Verwaltung-der-Landeshauptstadt-Hannover/Gleichstellungsbeauf\%C2 $\% A D t r a g t e-d e r-L a n d e s h a u p t s t a d t-H a n n o v e r / A k t u e l l e s / N e u e-R e g e l u n g-f u ̈ r-g e s c h l e c h t e r g$ erechte-Sprache [27.05.2020].

Vorschläge für einen geschlechtersensiblen Sprachgebrauch, Universität Freiburg (2018): gleichstellungsbuero.uni-freiburg.de/download/Uni_FlyerGBLeitlinie15_E1_DL_6Seiter _Print_CMYK_CS52.pdf [27.05.2020].

Wahlplattform Grüne Schweiz 2019-2023: gruene.ch/wp-content/uploads/2019/05/190112_ wahlplattform_d.pdf) [14.07.2020].

WDR 5 Funkhausgespräch vom 13.06.2019: Das Gendersternchen - Unfug oder Fortschritt? web.archive.org/web/20190702031123/https://www1.wdr.de/radio/wdr5/sendungen/funkh ausgespraeche/funkhausgespraeche1676.html [14.07.2020].

Wetschanow, Karin (2017): „Von nicht-sexistischem Sprachgebrauch zu fairen W_ortungen Ein Streifzug durch die Welt der Leitfäden zu sprachlicher Gleichbehandlung“. OBST 90: Sprache und Geschlecht. Band 1: Sprachpolitiken und Grammatik: 33-61.

Zifonun, Gisela (2018): Die demokratische Pflicht und das Sprachsystem: Erneute Diskussion um einen geschlechtergerechten Sprachgebrauch. Sprachreport 34: 44-56. 